\title{
Análisis de Sustentabilidad del Empleo de BCS para Calor de Proceso
}

\section{Sustainability Analysis of Aplication Solid Biofuels in Industrial Processes}

\author{
DURAN-GARCÍA, María D.†* † $^{*}$ WEBER, Bernd y JIMENEZ-GARCÍA, Juan A. \\ Universidad Autónoma del Estado de México, Facultad de Ingeniería \\ ID 1 Autor: María D. Duran-García / ORC ID: 0000-0003-3584-4783, Researcher ID Thomson: J-9111-2013 \\ ID $1^{\text {er }}$ Coautor: Bernd, Weber / ORC ID: 0000-000-0125-841X \\ ID $2^{\text {do }}$ Coautor: Juan A. Jimenez-García / ORC ID: 0000-0003-3584-4783, Researcher ID Thomson: J-9111-2013
}

DOI: $10.35429 / J U S D .2019 .17 .5 .1 .12$

Recibido: 30 de Septiembre, 2019; Aceptado 20 de Diciembre, 2019

\begin{abstract}
Resumen
El uso de biocombustibles sólidos en sistemas de combustión para la generación de calor industrial ha ganado interés en varios países del mundo, debido a las ventajas económicas que se ofrece su uso y a que se considera una fuente de energía renovable neutral de emisiones de gases de efecto invernadero. Aún con la gran disponibilidad del recurso biomásico en México con características apropiadas para la incineración, su desarrollo ha quedado rezagado en comparación a otros países. Por lo tanto es importante realizar estudios de viabilidad económica y ambiental de los sistemas ya instalado para identificar las oportunidades de optimización de los mismos. El presente artículo se enfoca en 2 estudios de viabilidad ambiental y económica del empleo de BCS para calor de proceso; empleando residuos de agave y astilla entre otros biocombustibles. Se realiza un análisis de emisiones, un análisis ACV así como un análisis de factibilidad económica y se comparan los resultados con el uso de combustibles fósiles para determinar su factibilidad en un mediano plazo. El presente artículo se enmarca en los resultados obtenidos en las 3 primeras etapas del proyecto CEMIEBIO SOLIDOS. (PROYECTO SENER-CONACYT).
\end{abstract}

\begin{abstract}
The use of solid biofuels in for industrial heat has gained interest in several countries of the world, due to the economic advantages offered by its use since it is considered a neutral renewable energy source of greenhouse gas emissions greenhouse. Even with the high availability of biomass in Mexico, with appropriate characteristics for incineration, its development has lagged behind other countries. It is important to carry out economic and environmental feasibility studies of the biomass systems already installed in order to identify opportunities for its optimization. This article presents 2 environmental and economic feasibility studies of the use of BCS for process heat; using agave bagasse and pine woodchip among other biofuels. An emission analysis, an ACV analysis as well as an economic feasibility analysis are carried out and the results are compared with the use of fossil fuels to determine their feasibility in the medium term.
\end{abstract}

Solid Biofuels, Sustainability

Citación: DURAN-GARCÍA, María D., WEBER, Bernd y JIMENEZ-GARCÍA, Juan A. Análisis de Sustentabilidad del Empleo de BCS para Calor de Proceso. Revista del Desarrollo Urbano y Sustentable. 2019. 5-17: 1-12

\footnotetext{
*Correspondencia al autor (correo electrónico: mddg_2210@ hotmail.com)

$\dagger$ Investigador contribuyendo como primer autor
} 


\section{Introducción}

Actualmente los combustibles fósiles como el petróleo y gas natural representan la principal fuente de energía a nivel mundial, sin embargo, estas fuentes comenzarán a escasear en los próximos 40-50 años; esto aunado a todos los daños ambientales que ocasiona el uso de estas fuentes de energía hace urgente el cambio hacia un sistema energético más sustentable.

En este sentido, las fuentes renovables de energía constituyen una excelente alternativa para combatir el cambio climático, el deterioro ambiental y la, cada vez más inminente, escases de recursos ocasionada por el actual sistema energético, basado en combustibles fósiles.

De entre las fuentes renovables de energía la biomasa constituye una de las opciones más viables. Esta, contribuye entre un 10 y un $15 \%$ de la demanda de energía global.(Oregon, 2010; Demirbas,2005).

Específicamente los biocombustibles sólidos fueron la primera bioenergía empleada en México, principalmente en aplicaciones domésticas. Sin embargo, a finales del siglo XX se le ve como una opción que apoya transición energética, esto debido a la inseguridad de los combustibles fósiles, los altos costos y nivel de emisiones de los mismos.

Los biocombustibles sólidos (BCS) comprenden desde la leña y carbón natural, hasta bagazo, aserrín y residuos agrícolas y sólidos municipales. Estos pueden utilizarse con la tecnología de combustión directa para la generación de calor, electricidad o cogeneración a mediana y gran escala. Algunas de estas materias primas requieren tratamientos previos como reducción de tamaño, secado o transformación a pellets.

Entre los principales residuos forestales y agrícolas que pueden aprovecharse para suministrar calor a procesos están los rastrojos, residuos de palma, caña y agave. Este tipo de biomasa puede ser utilizada sin procesamiento previo. Sin embargo, en su estado natural tiene alto contenido de humedad $(50 \%$ en el bagazo, $20 \%$ a $50 \%$ en el aserrín, $20 \%$ a $45 \%$ en la leña) además, presenta una baja densidad $0.15 \mathrm{t} / \mathrm{m}^{3}$ el bagazo, $0.12 \mathrm{t} / \mathrm{m}^{3}$ el aserrín y 0.3 a $0.5 \mathrm{t} / \mathrm{m}^{3}$ la leña.
Así pues, algunas de ellas precisan un procesamiento de la biomasa que incluye su secado y densificación, estos procesos incrementan su costo, lo que aunado al transporte y almacenaje hace en algunos casos poco atractivo su uso como energético principalmente en el sector industrial. (Koh,2003).

No obstante, el poco uso de la biomasa a nivel industrial es más bien resultado de la poca información acerca de la disponibilidad de la misma en el territorio nacional, de sus costos de procesamiento y de sus propiedades caloríficas. En este sentido existen proyectos como el cluster de biocombustibles sólidos, proyecto auspiciado por el fondo de Sustentabilidad Energética SENER-CONACYT que estan enfocados a identificar la disponibilidad de biomasa en diferentes regiones del país y caracterizarla para su uso en el sector residencia, industrial y producción de energía eléctrica.

Por otro lado, gran mayoría de los procesos en la industria, en este País y en el mundo requieren producción de energía térmica, de procesos y vapor; en este sentido a nivel mundial el porcentaje de energía primaria que se emplea para procesos industriales es de aproximadamente el $30 \%$., mientras que el resto se emplea en energía eléctrica y transporte (IEA, WEO, 2018). En lo que respecta a nuestro País, de acuerdo con el Balance Nacional de Energía (2018) el 62\% de la energía primaria se consume por procesos industriales, esto es un porcentaje importante más si se considera que gran parte de esta energía se emplea como calor de proceso o generación de vapor y que los biocombustibles sólidos podrían emplearse para la producción de esta energía, mediante quema directa.

Respecto a los BCS utilizados muchas veces la industria los considera como residuo sin costo alguno, y por eso existe poco interés sobre la eficiencia de la caldera. Sin embargo, en la evaluación se debe de asignar un valor a los BCS con fines de mostrar que cantidad de "dinero" que se expulsa por la chimenea. Así el BCS debe de tener un valor que varía entre precio regional y global dependiendo del tamaño de la industria del caso específico. 
A nivel mundial existen numerosos proyectos que muestran la factibilidad del empleo de biocombustibles sólidos para producción de vapor de proceso. Esto se debe a que la quema directa de biomasa es mucho más económica debido a que, generalmente no se requiere ningún proceso adicional. De acuerdo con el (IEA Bioenergy, 2013) en el 2009 el uso de la biomasa para procesos industriales alcanzó los 7 EJ por año, y los 10 países que emplean la mayor cantidad de biomasa en procesos industriales se enlistan en la tabla 1.

No. País
\begin{tabular}{|l|l|r|}
\multicolumn{2}{c}{ Consumo de BCS } \\
PJ \\
\hline 1 & Brasil & 1317 \\
\hline 2 & India & 1195 \\
\hline 3 & Estados Unidos & 1063 \\
\hline 4 & Nigeria & 379 \\
\hline 5 & Canadá & 287 \\
\hline 6 & Tailandia & 283 \\
\hline 7 & Indonesia & 272 \\
\hline 8 & República Democrática del Congo & 185 \\
\hline 9 & Suecia & 169 \\
\hline 10 & Paquistán & 135 \\
\hline
\end{tabular}

Tabla 1 Diez países con el mayor uso de biomasa sólida en el sector industrial en el año 2009

Fuente: IEA Bioenergy 2013

Por su parte en México, si bien existen algunos proyectos exitosos en los cuales se emplea biomasa como combustible, poco se conoce acerca de su factibilidad económica y sobre su sustentabilidad. Esto hace que empresas que tienen disponibilidad de residuo lo pueden emplear como BCS lo descarten inmediatamente. Existen diferentes estudios de factibilidad del uso de biomasa sólida, pero la mayoría son a nivel internacional (Demirbas, 2001).

Dado lo anterior en el presente trabajo se analiza la factibilidad técnica y la sustentabilidad del empleo de biocombustibles sólidos en procesos industriales. El análisis se realiza bajo dos criterios: el análisis de sustentabilidad, tomando como base el análisis de ciclo de vida y el análisis económico, considerando el ahorro obtenido al dejar de comprar un combustible fósil.

El aporte principal de este artículo es dar a conocer la factibilidad técnica y sustentabilidad del empleo de BCS en procesos industriales.
Los trabajos publicados en este sentido, muestran únicamente el análisis de sustentabilidad o un análisis económico, pero sin considerar costos reales de los equipos y de su mantenimiento Demirbas A. (2005).

Como se indicó anteriormente este trabajo está enmarcado en el proyecto Cluster de biocombustibles sólidos, desarrollado por la línea 5 enfocada al uso de biomasa en la industria. Así pues, considera análisis de casos reales.

Particularmente se realiza el análisis de dos casos de éxito, uno en la industria tequilera y otro en la industria del café. Es importante mencionar que no se presentan los costos del producto ni precios de venta en cada caso, dado que se ha firmado un convenio de confidencialidad con cada empresa; sin embargo, si se consideran costos reales para realizar el análisis económico.

\section{Metodología}

Como se indicó anteriormente, en el presente trabajo se analiza la factibilidad del empleo de BCS bajo dos criterios: sustentabilidad y factibilidad económica. El análisis se realiza para los dos casos en los cuales se lleva a cabo la comparación entre el empleo de un combustible fósil y el empleo de un BCS.

Primeramente se realiza el análisis de sustentabilidad bajo la perspectiva del ACV, pero enfocándose solamente a la generación de energía térmica en la caldera. Posteriormente se realiza el análisis económico considerando como flujo de caja la diferencia de costo entre el combustible fósil y el BCS. En los siguientes subapartados se describe con mayor claridad la metodología aplicada.

\section{Análisis de ciclo de vida}

El Análisis de Ciclo de Vida (ACV) es una técnica de gestión ambiental desarrollada para analizar los aspectos e impactos ambientales potenciales relativos a productos tanto manufacturados como consumidos (ISO, 2006). 
El Ciclo de Vida de un producto involucra flujos de materia, energía y dinero a lo largo de su cadena de valor, no obstante, para tener un panorama completo se deben considerar los impactos de todos los actores: trabajadores, comunidades locales, consumidores y la sociedad en general (Garcia, 2019) involucrados con la producción y consumo del producto (UNEP, 2011; Rodríguez, et al.2019.).

El ACV considera aspectos e impactos ambientales potenciales tales como el uso de recursos y las consecuencias ambientales de las emisiones causadas a lo largo de todo el ciclo de vida de un producto (ISO, 2006). Esta técnica se desarrolla en cuatro fases normalmente interdependientes entre sí:

Definición del objetivo y el alcance. A partir del uso que tendrá el estudio se plantean el objetivo y el alcance, con lo que se podrá establecer la profundidad, amplitud y nivel de detalle del ACV, así como la delimitación del sistema a estudiar. En este proyecto en particular solamente se limita al análisis de la sustentabilidad del empleo de combustible fósil y BCS.

Análisis de inventario del ciclo de vida (ICV). En esta fase se recopilan aquellos datos de flujos de entrada/salida en el sistema estudiado que proporcionen información necesaria para cumplir con el objetivo definido (ISO, 2006). Los flujos requeridos para el inventario se ejemplifican de manera gráfica por la UNEP (2011) en la figura 1. Y para el caso particular que aquí se presenta en la figura 2.

- Evaluación del impacto ambiental del ciclo de vida (EICV). Proporciona información adicional para el análisis de los resultados del ACV, al relacionar estos últimos con indicadores de índole ambiental (como cambio climático, uso de suelo, uso de agua, efectos tóxicos al hombre, agotamiento de ozono, acidificación, biodiversidad, etc.)

Interpretación del ciclo de vida. Es la fase final del $\mathrm{ACV}$, en ella se resumen $\mathrm{y}$ discuten los resultados $\mathrm{y}$ se toman las decisiones pertinentes.
En el presente artículo se aplica la metodología de análisis de ciclo de vida para analizar la aplicación de BCS en la producción de energía térmica para un proceso industrial. Es importante destacar que no se pretende analizar el ciclo de vida del producto final, ni de la cadena completa, sino de la producción de energía térmica comparando dos escenarios:

- Producción de energía térmica empleando combustible fósil, gas natural o combustóleo, según el caso

Producción de energía térmica empleando BCS, siendo parte de este biocombustible el propio residuo del proceso productivo.

La metodología de ACV puede aplicarse de manera particular a los parámetros o indicadores que se requiera estudiar; así pues, en el presente trabajo se aplica el análisis a los impactos de los escenarios indicados, considerando tres categorías de impacto que son:

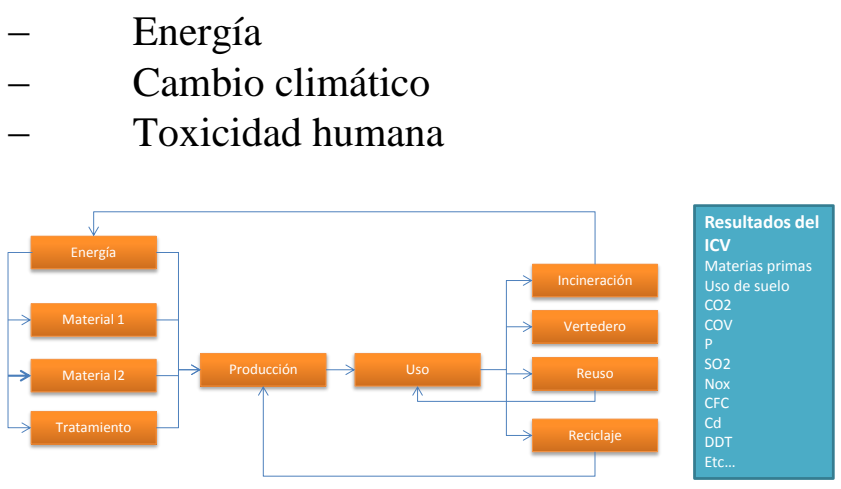

Figura 1 Flujos de información necesarios para el inventario de ciclo de vida

Fuente: UNEP, 2011

En el apartado 3 se presenta la descripción precisa de los casos y escenarios analizados. Es importante destacar que en ambos casos analizados se desconocen datos como el número de trabajadores involucrados con el proceso, la producción mensual, el costo de materia prima, entre otros, por lo que no es posible realizar un ACV completo del producto; además, el objetivo es analizar la sustentabilidad del empleo de BCS por lo que no se considera necesario realizar un análisis completo. Con respecto a la unidad funcional considerada para el análisis esta es 1 GJ de energía térmica, con lo cual se analiza el impacto ambiental y económico de la producción de esta unidad funcional. Esta unidad funcional se muestra en la figura 2 . 


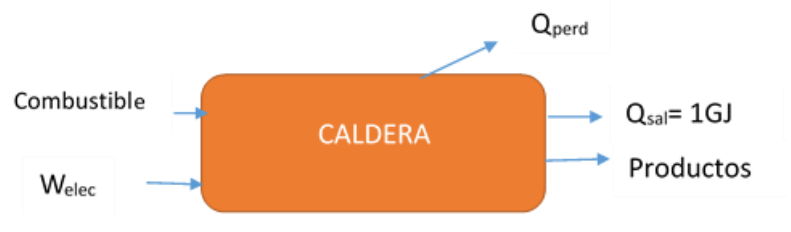

Figura 2 Unidad funcional analizada Fuente: Elaboración Propia

Respecto a los factores de emisión de cada componente, estos se determinaron con base en la guía para el inventario de gases efecto invernadero y se muestran en la tabla 2. Y fueron obtenidos de la guía nacional de gases de efecto invernadero (IPCC,2006).

\begin{tabular}{|l|r|r|r|}
\hline Combustible & \multicolumn{1}{|c|}{$\mathbf{C O}_{2}$} & \multicolumn{1}{|c|}{$\mathbf{C H}_{4}$} & \multicolumn{1}{|c|}{$\mathbf{N}_{\mathbf{2}} \mathbf{O}$} \\
\hline & {$\left[\mathrm{kg} \mathrm{CO}_{2} / \mathrm{TJ}\right]$} & {$\left[\mathrm{kg} \mathrm{CH}_{4} / \mathrm{TJ}\right]$} & {$\left[\mathrm{kg} \mathrm{N}_{2} \mathrm{O} / \mathrm{TJ}\right]$} \\
\hline Carbón & 94600 & 1 & 1.5 \\
\hline Diésel & 74100 & 3 & 0.6 \\
\hline Combustóleo & 77400 & 3 & 0.6 \\
\hline Gas Natural & 56100 & 1 & 0.1 \\
\hline
\end{tabular}

Tabla 2. Factores de emisión por tipo de combustible Fuente: IPCC, 2006

\section{Análisis económico}

Con respecto al análisis económico, este se realiza con el objetivo de determinar la viabilidad técnico-económica del empleo de BCS en los casos analizados. Así pues, se analiza del costo producción de energía térmica (vapor) en los escenarios indicados en siguiente apartado. Es importante destacar que si bien existen algunos trabajos enfocados al análisis de factibilidad del empleo de BCS (Walker, 2009) no realizan el análisis con costos y datos reales de los sistemas.

Se consideró como base la metodología propuesta por Keenan J. y Maguire M. (1983) y el criterio de factibilidad económica se basa en la relación de costos determinados para ambas calderas analizadas (la de biomasa y la de combustible fósil), sin embargo, ambas soluciones tienen diferentes costos de inversión y de operación. Así pues, con los datos económicos de ambas opciones se realiza el análisis de distribución de costos y se determina el flujo de caja como la diferencia entre los costos totales empleando combustible fósil y empleando BCS.

$B=C_{T^{\prime} t_{C F}-} C_{T o t_{B C S}}$
Donde:

$C_{T o t_{C F}}=$ Costo total de generación con combustible fósil.

$C_{T o t_{B C S}}=$ Costo total de generación con BCS.

El costo total, $C_{T o t}$ en la ecuación 1 incluye la totalidad de los gastos operacionales en que se debe incurrir para llevar a cabo la producción de energía, incluyendo costos de operación, costo de mantenimiento, costo de energía eléctrica y de transporte de combustible, entre otros, es decir:

$C_{T o t}=C_{T c}+C_{o m}+C_{a}$

Donde:

$C_{T c}=$ Costo total de combustible

$C_{o m}=$ Costo de operación y mantenimiento

$C_{a}=$ Costo de amortización

Con base en el flujo de caja determinado mediante la ecuación 1 se obtiene el tiempo de retorno de inversión, para analizar la factibilidad del empleo del BCS, que es la caldera con mayor costo de inversión.

Es importante destacar que en este trabajo no se describe información específica de algunos costos de operación y de adquisición de las calderas por tratarse de información confidencial por parte de la empresa analizada y el fabricante de calderas. Particularmente analiza el cambio de combustible y el empleo de una caldera de biocombustible en lugar de una de combustible fósil.

\section{Casos analizados}

Primeramente, se realizó un análisis de algunas empresas que emplean calderas pirotubulares, o acuatubulares de media potencia; de entre ellas se detectaron algunas empresas que emplean biocombustible sólido o que tienen potencial para emplearlo, entre ellas se determinaron los casos de éxito en los cuales se contaba con información suficiente para realizar el análisis de factibilidad. Se seleccionaron aquellas empresas que emplean actualmente una caldera de biomasa y que también contaban con alguna instalación de combustible fósil (combustóleo o gas natural) con el fin de analizar los dos escenarios: empleo de BCS y empleo de combustible fósil. En los subapartados siguientes se describen estos casos. 


\section{Bagazo de agave}

Empresa productora de Tequila ubicada en Jalisco, la cual emplea bagazo de agave, mezclado con otros biocombustibles como astilla; además de biogás (producido por la misma empresa) en una segunda etapa de combustión en una caldera pirotubular hibrida (acua-pirotubular) de 800BHP.

La caldera es de piso oscilante y emplea como combustible 45 toneladas de biomasa por día, operando 24 horas a plena carga. El vapor generado en la caldera se emplea para los hornos de cocimiento de las piñas, para los alambiques, para el proceso de hidrólisis y destilación.

El vapor producido en la caldera es vapor sobrecalentado a 95 libras de presión y una temperatura de $178^{\circ} \mathrm{C}$, con un flujo de vapor de $13676 \mathrm{~kg} / \mathrm{h}$.

Como se indicó, la caldera emplea BCS y biogás como combustible; en los siguientes porcentajes $15 \%$ biogás producido en el biodigestor empleado para el tratamiento de las vinazas y $85 \%$ BCS compuesto de la siguiente manera $70 \%$ de bagazo de agave con una humedad del $60.6 \%$ y $30 \%$ de astilla de madera de pino o de tarima, con una humedad entre el 15 y el 22\% (Datos analizados en laboratorio).

La tabla 3 muestra el valor del poder calorífico del combustible descrito. Considerando ambas opciones $100 \%$ del BCS como bagazo de agave o $70 \%$ bagazo de agave y $30 \%$ astilla de pino. El poder calorífico inferior (PCI) el que se deberá asumir como referencia ya que el vapor de agua presente en los gases de combustión nunca se lleva hasta la condensación.

Se considera analizar el caso de que la empresa empleara el 100\% de bagazo de agave, aunque este caso de momento no es factible ya que la empresa no tiene el suficiente residuo de bagazo para toda su demanda energética.

\begin{tabular}{|l|r|r|}
\hline \multicolumn{1}{|c|}{ Combustible } & \multicolumn{1}{c|}{ PCS } & \multicolumn{1}{c|}{ PCI } \\
\hline & {$[\mathbf{M J} / \mathbf{k g}]$} & {$[\mathbf{M J} / \mathbf{k g}]$} \\
\hline Bagazo de Agave & 9.6338 & 7.7246 \\
\hline Mezcla 70/30 & 9.414 & 7.488 \\
\hline
\end{tabular}

Tabla 3. Poder calorífico base del combustible Fuente: Elaboración Propia
Con respecto al biogás, sólo se tiene la fracción de metano y el flujo energético, que se presentan en la tabla 4 , no se conoce la composición elemental del mismo por lo que no se considera durante el análisis emisiones pero si en el análisis económico.

\begin{tabular}{|l|r|}
\hline \multicolumn{1}{|c|}{ Biogas } & \multicolumn{1}{c|}{ Valores } \\
\hline Flujo & $298.93 \mathrm{~kg} / \mathrm{hr}$ \\
\hline Flujo energético & $4575337 \mathrm{~kJ} / \mathrm{h}$ \\
\hline Fracción metano & 52.41 \\
\hline
\end{tabular}

Tabla 4 Características del biogás

Fuente: Empresa.

La empresa analizada empleaba anteriormente una caldera de combustóleo de $1000 \mathrm{HP}$, por lo que el escenario comparativo que se consideró fue éste.

\section{Bagazo de Café}

Empresa de café soluble ubicada en el Estado de México, emplea bagazo de café, combinado con arena sílice, para producir el $50 \%$ de sus necesidades de vapor en una caldera acuatubular de lecho fluidizado, con capacidad de $33 \mathrm{MW}$ útil, que produce vapor saturado a 17.4 bar.

El lecho fluidizado se compone principalmente de arena sílice con total de $40 \mathrm{t}$. El otro $50 \%$ de la energía requerida por la empresa se genera con una caldera de gas natural de $17 \mathrm{MW}$. El bagazo de café es parecido a una biomasa torrificada debido al tratamiento térmico al que fue sometido sus características de poder calorífico y humedad se muestran en la tabla 5 .

\begin{tabular}{|l||r|r|r|}
\hline \multicolumn{1}{|c|}{ Biomasa } & Humedad & $\begin{array}{c}\text { PCI } \\
\text { MJ/kg } \\
\text { ("as } \\
\text { fired") }\end{array}$ & $\begin{array}{c}\text { Cenizas } \\
\text { \% en } \\
\text { (masa seca) }\end{array}$ \\
\hline $\begin{array}{l}\text { Bagazo torrificado } \\
\text { y prensado }\end{array}$ & 58.6 & 12.5 & 2 \\
\hline Cascarilla & 19.7 & 17.3 & 6.3 \\
\hline Lodos & 90.9 & 2.5 & 1.6 \\
\hline
\end{tabular}

Tabla 5. Características de los combustibles empleados en la caldera de lecho fluidizado

Fuente: Elaboración Propia

El tratamiento térmico, que actúa como una extracción, reduce el contenido de minerales de la biomasa y además incrementa la temperatura de fundición de cenizas, por extraer los minerales más solubles. 
Lamentablemente la biomasa se obtiene en el proceso con una humedad mayor del $70 \%$ y a través de la etapa de deshidratación mecánica en tornillos prensa se logra de reducir la humedad a cerca del $60 \%$. Junto con el bagazo se incineran en la caldera los lodos derivados de la planta de tratamiento de aguas residuales. En conjunto el lodo y la cascarilla forman un material de alta humedad igual que el bagazo de café. Sus características también se indican en la tabla 5 .

Como estrategia energética se pretende de requerir cada vez menos la aportación de la combustión de combustibles fósiles. Así el análisis está definido en evaluar el uso de combustible alterno adicional, que recibirá la planta productiva, con el fin de generar el $100 \%$ de la energía requerida con la caldera de biomasa. El combustible considerado es pellet de pino con las siguientes características.

\begin{tabular}{|l|c|r|}
\hline Biomasa & $\begin{array}{c}\text { PCI } \\
\text { MJ/kg } \\
\text { ("as fired") }\end{array}$ & \multicolumn{2}{c|}{$\begin{array}{c}\text { Cenizas } \\
\text { en (masa seca) }\end{array}$} \\
\hline Pellet de pino & 19.5 & 2.69 \\
\hline
\end{tabular}

Tabla 6 Características del pellet de pino Fuente: Elaboración Propia

\section{Resultados}

\section{Bagazo de agave}

Es muy importante recalcar que el uso de biomasa residual como combustible ayuda a la no-generación de residuos sólidos cuyo factor de contaminación puede llegar a ser muy alto si su disposición no es la adecuada, lo cual sucede muy a menudo.

\section{a) Emisiones}

Con respecto a las emisiones de gases de efecto invernadero, la tabla 7 muestra los valores de toneladas de dióxido de carbono equivalentes que se obtendrían con la combustión de la composición $70 \%$ bagazo de agave y $30 \%$ astilla de madera, con la caldera de 800BHP considerada para el análisis. Para realizar estos cálculos se consideró un tiempo de operación reportado en el apartado 3 .

\begin{tabular}{|c|c|c|c|c|}
\hline $\begin{array}{c}\text { Emisiones } \\
\text { estimadas } \\
\text { Con bagazo } \\
\text { de agave }\end{array}$ & $\begin{array}{l}\text { Razón } \\
\text { [mg/kJ] }\end{array}$ & $\begin{array}{l}\text { Flujo } \\
\text { kg/h }\end{array}$ & $\begin{array}{c}\text { Conc } \\
\mathrm{mg} / \mathrm{m}^{3}{ }_{\text {normal }}\end{array}$ & $\begin{array}{l}\text { AR4 PCG* } \\
\text { tCO } \\
\text { ee/año }\end{array}$ \\
\hline $\begin{array}{l}\text { PM-10 con } \\
\text { multiciclón }\end{array}$ & 0.1161 & 5.58 & 210 & $\mathrm{~s} / \mathrm{d}$ \\
\hline $\begin{array}{l}\text { PM-2.5 con } \\
\text { multicilón }\end{array}$ & 0.0688 & 3.30 & 125 & $\mathrm{~s} / \mathrm{d}$ \\
\hline Total de PM & 0.1290 & 6.19 & 234 & $\mathrm{~s} / \mathrm{d}$ \\
\hline NOx & 0.0946 & 4.45 & 171 & 6,454 \\
\hline $\mathrm{CO}$ & 0.2580 & 4.13 & 467 & 20 \\
\hline $\begin{array}{l}\text { Compuestos } \\
\text { orgánicos } \\
\text { volátiles } \\
\text { (VOC) }\end{array}$ & 0.0073 & 0.35 & 13 & 0 \\
\hline $\begin{array}{l}\text { Carbono } \\
\text { orgánico } \\
\text { total (TOC) }\end{array}$ & 0.0168 & 0.81 & 30 & 0 \\
\hline \multicolumn{4}{|c|}{ Emisiones por consumo de electricidad } & 291 \\
\hline
\end{tabular}

Tabla 7 Potencial de gas de efecto invernadero para las emisiones a partir de mezcla $70 \%$ bagazo de agave y $30 \%$ astilla de pino

Fuente: Empresa fabricante de calderas

Con base en los datos de la tabla anterior y considerando los indicados en la tabla 2. Se realizó el análisis de emisiones de $\mathrm{CO}_{2}$ equivalente considerando el potencial de calentamiento global y los factores de emisión del programa GEI México. Los resultados se muestran en la gráfica de la figura 3.

Como puede observarse, las emisiones de $\mathrm{CO}_{2}$ correspondientes al combustóleo son mucho más altas que aquellas que presenta la combustión de bagazo de agave y la mezcla de bagazo y astilla de pino. Además, debe considerarse que las emisiones de $\mathrm{CO}_{2}$ son neutrales siempre y cuando el recurso sea obtenido de cultivos sustentables.

\section{Emisiones de tCO2e/GJ}

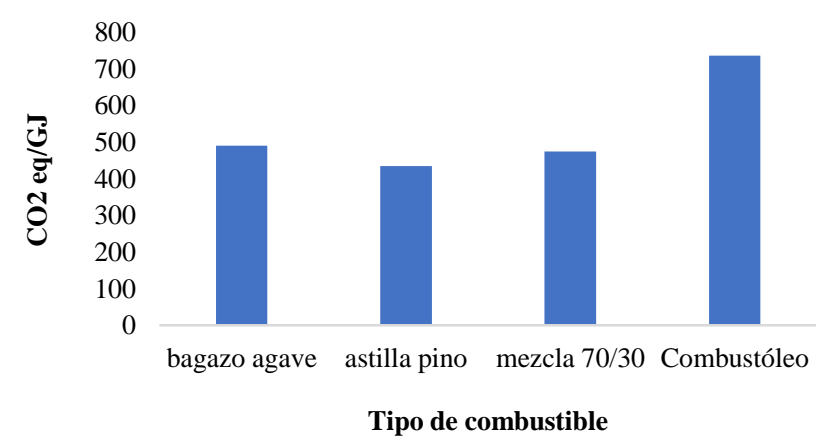

Figura 3 Emisiones de $\mathrm{CO}_{2}$ equivalente por tipo de combustible analizado (5) 
b) Costo de generación.

Para determinar los costos de operación se consideraron costos de combustible, mano de obra de operadores, mantenimiento y disposición de residuos entre otros. Particularmente, el costo del combustóleo se obtuvo a partir de información de PEMEX (2018). Mientras que el costo de la astilla de pino fue proporcionado por la empresa. Estos costos se muestran en la tabla 8. Para el bagazo de agave no se considera ningún costo porque este es residuo de la propia empresa.

$$
\begin{array}{|l|c|}
\hline \text { Combustible } & \text { Costo } \\
\hline \text { Astilla }^{1} & \$ 2.00 / \mathrm{kg} \\
\hline \text { Combustóleo } & \$ 7.12 / \text { litro } \\
\hline
\end{array}
$$

Tabla 8. Costo total del combustible Fuente: PEMEX, 2018

Considerando estos costos, e incluyendo los costos de energía eléctrica, en la gráfica de la figura 4 se muestra la comparación en costo de generación empleando como unidad funcional el GJ, es decir se obtiene el costo por cada GJ generado en cada uno de los tres casos.

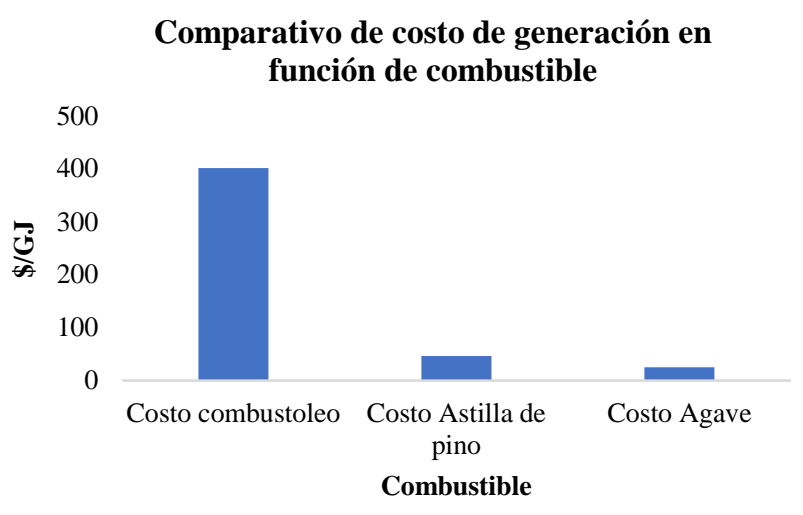

Figura 4 Costo de generación de 1GJ con los diferentes tipos de combustible analizados

Como puede observarse en la gráfica el costo de generación de energía, empleando combustóleo como combustible es 5 veces mayor que si se emplea combustible.

Finalmente, se realiza un análisis del flujo de caja y el retorno de la inversión, con base en la metodología descrita en el apartado 2.2. considerando una depreciación de la caldera por un periodo de 10 años.
Flujo acumulado neto de efectivo mezcla

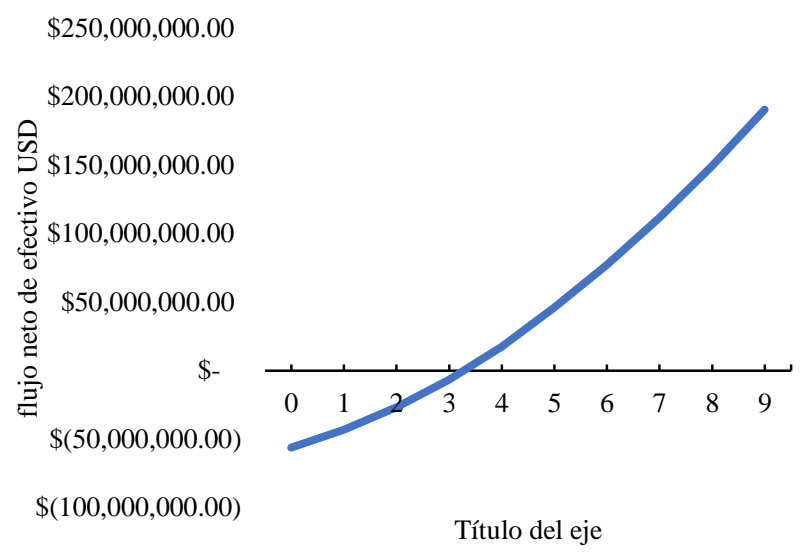

Figura 5. Flujo neto de efectivo derivado del ahorro en consumo de combustible al emplear una caldera de biomasa. (70\% Bagazo y $30 \%$ astilla)

Fuente: Elaboración Propia

Flujo acumulado neto de efectivo de $100 \%$ gabazo

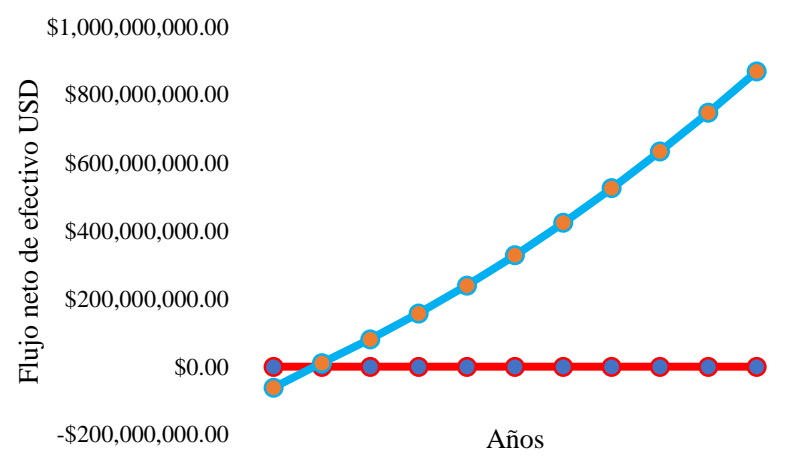

Figura 6 Flujo neto de efectivo derivado del ahorro en consumo de combustible al emplear una caldera de biomasa. (100\% bagazo)

Fuente: Elaboración Propia

Con base en estas consideraciones se obtuvo el periodo de retorno de la inversión para determinar la factibilidad del proyecto. Así pues, en la gráfica de la figura 5 y 6 se muestra el flujo acumulado de efectivo en función del tiempo para ambos escenarios: $70 \%$ bagazo $+30 \%$ astilla y $100 \%$ bagazo.

\section{Bagazo de café}

Es evidente que, dada la naturaleza del sistema a analizar, el residuo, que la planta misma genera, es económicamente el combustible más viable, porque no tiene ningún costo. Pero si el cliente adquiere combustible alterno se debe de considerar el costo de adquisición. Como muestra la tabla 9 el gas natural es de menor costo, por lo cual no se llega a una amortización al momento de invertir en un proyecto de incrementar el factor de planta de la caldera de biocombustibles sólidos. 
Combustible Costo unitario Costo anual \begin{tabular}{|l|r|r|}
\hline Bagazo de café & 0 & 0 \\
\hline Pellet $^{1}$ & $\$ 4.00 / \mathrm{kg}$ & $\$ 93.3$ millones \\
\hline Gas Natural & $\$ 78.9646 / \mathrm{GJ}$ & $\$ 31.5$ millones \\
\hline
\end{tabular} \begin{tabular}{|l|l|l|}
\hline Gas Natural & $\$ 78.9646 / \mathrm{GJ}$ & $\$ 31$ \\
\hline
\end{tabular}

Tabla 9: Costo de los combustibles Fuente: Elaboración propia con base en PEMEX, 2018.

\section{a) Emisiones}

La energía térmica generada para ambos procesos es de $3.9910^{5} \mathrm{GJ}$ y representa un flujo másico de los escapes secos de 48600 toneladas al año para el gas natural y 107300 toneladas para el pellet de piño. Con la concentración permisible de la NOM-085 ambos escenarios emitirían los diferentes contaminantes según de la siguiente tabla.

\begin{tabular}{|c|c|c|c|c|}
\hline Escenario & Partículas & SO2 & $\begin{array}{c}\text { NO } \\
\mathbf{x}\end{array}$ & $\mathbf{C O}$ \\
\hline \multicolumn{5}{|l|}{ t/año } \\
\hline Gas Natural & & & 62.5 & 159.2 \\
\hline Pellet de Pino & 56.3 & 187.7 & 138.1 & 351.6 \\
\hline Diferencia & 56.3 & 187.7 & 75.6 & 192.4 \\
\hline
\end{tabular}

Tabla 10 Emisión de contaminantes de los escenarios de la combustión de gas natural y pellet de pino Fuente: Elaboración Propia

\section{b) Efecto invernadero}

La emisión de gases de efecto invernadero considera que la combustión del pellet de pino libera $\mathrm{CO}_{2}$ neutral, lo que es válido mientras que la obtención del recurso de madera se derive de un cultivo sustentable. Los resultados se muestran en la tabla 11 donde se observa que sólo el gas natural emite gases de efecto invernadero de manera directa. De manera indirecta lo que es la emisión ocasionada para modificar y llevar el combustible al sitio el pellet de pino tiene una mayor hulla de carbono que el gas natural. Sin embargo por la implementación de la combustión de pellet de pino se logra una reducción de gases de efecto invernadero de 15737 toneladas al año.

\begin{tabular}{|l|r|r|r|}
\hline \multicolumn{1}{|c|}{ Escenario } & \multicolumn{2}{|c|}{ Directo Indirecto } & \multicolumn{1}{c|}{ Total } \\
\hline & \multicolumn{1}{|c|}{ t/año } \\
\hline Gas Natural & 21223 & 858 & 22081 \\
\hline $\begin{array}{l}\text { Pellet de } \\
\text { Pino }\end{array}$ & 0 & 6344 & 6344 \\
\hline Diferencia & 21223 & -5486 & 15737 \\
\hline
\end{tabular}

Tabla 11. Emisión de gases de efecto invernadero en toneladas equivalentes de $\mathrm{CO}_{2}$ para los escenarios de la combustión de gas natural y pellet de pino

Fuente: Elaboración Propia
El balance de energía debe de equilibrar la demanda de energía primaria para la operación de la caldera con los dos diferentes combustibles seleccionados. El valor indirecto considera la energía primaria que se requiere para transformar y transportar los energéticos al sitio. Como la transformación y transporte para el pellet de pino es mayor se tiene a un incremento del consumo de energía primaria sin embargo el escenario del pellet de pino se basa en el uso de recursos renovables. Estos resultados se muestran en la tabla 12.

\begin{tabular}{|l|r|r|r|}
\hline \multicolumn{1}{|c|}{ Escenario } & \multicolumn{2}{c|}{ Directo Indirecto } & Total \\
\hline & TJ/año & \multicolumn{2}{c|}{} \\
\hline Gas Natural & 399 & 16 & 415 \\
\hline Pellet de pino & 399 & 103 & 502 \\
\hline Diferencia & 0 & -87 & -87 \\
\hline
\end{tabular}

Tabla 12 Demanda de energía primaria para la operación de las calderas

Fuente: Elaboración Propia

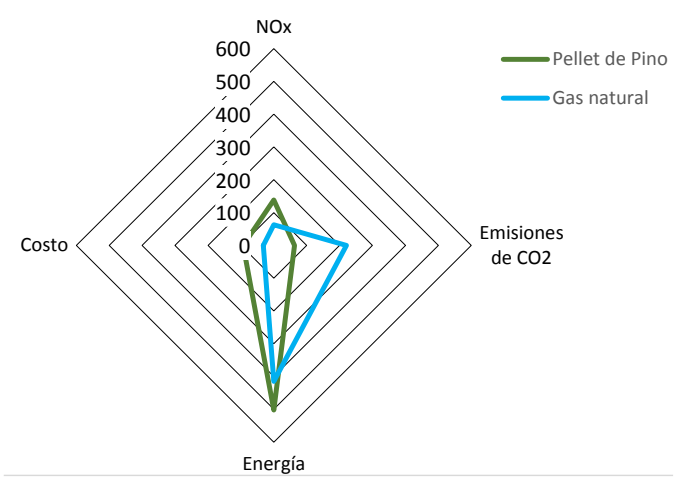

Figura 7 Gráfica de red los factores analizados: NOx, Emisiones, costo y energía, $\mathrm{CO} 2$ y $\mathrm{NOx}$ en t/año y energía en TJ/año

Fuente: Elaboración Propia

La figura 7, muestra en una gráfica de red los factores analizados en el presente estudio y se observa que la implementación de incinerar pellets incrementaría el costo de operación y la demanda de energía primaria y las emisiones de NOx, sin embargo disminuye las emisiones de $\mathrm{CO}_{2}$. Asimismo, en la gráfica de la figura 8 se muestra el análisis para la generación de $1 \mathrm{MJ}$ de energía.

De la misma forma que para el bagazo de agave, se realiza el análisis de flujo de caja para determinar el retorno de la inversión; este análisis se realiza considerando que el ingreso es la diferencia entre el costo de generación de vapor empleando BCS y empleando combustible fósil. 


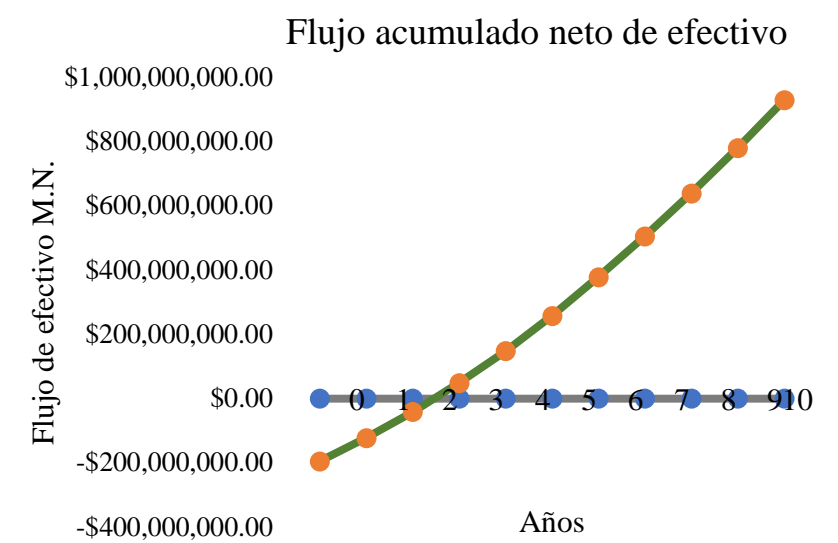

Figura 8 Flujo neto de efectivo derivado del ahorro en consumo de combustible al emplear una caldera de biomasa, considerando sólo bagazo de café

Fuente: Elaboración Propia

Con base en estas consideraciones se determinó el periodo de retorno de la inversión para observar la factibilidad del proyecto. Así, en la gráfica de la figura 8 se muestra el flujo acumulado neto de efectivo en función del tiempo, empleando únicamente bagazo de café; se observa que el flujo neto acumulado de efectivo es positivo a partir del 2 año considerando impuestos y depreciación.

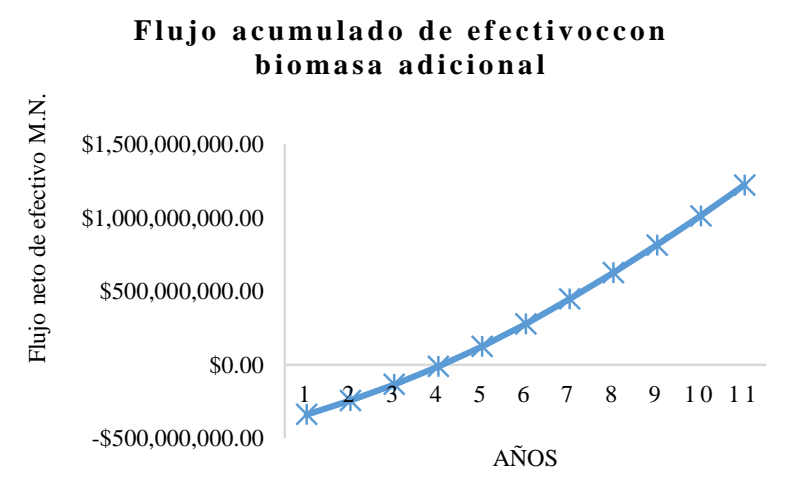

Figura 9 Flujo neto de efectivo derivado del ahorro en consumo de combustible al emplear una caldera de biomasa, considerando bagazo de café y pellet de pino. Fuente: Elaboración Propia

De igual manera se realizó considerando la estrategia energética descrita en el aparatado 3.2, es decir, considerando que la caldera de biomasa produce el $100 \%$ de la demanda energética de la planta mediante la compra de biomasa adicional, la cual es pellet de pino (50\% bagazo y $50 \%$ pellet), esta se muestra en la gráfica de la figura 9 y se observa que el periodo de retorno de inversión aumenta a casi 4 años debido al costo adicional que representa esta biomasa. Sin embargo, aun así es un proyecto factible.

\section{Conclusiones}

Los resultados muestran la factibilidad del empleo de biocombustibles sólidos para procesos térmicos, cuando se tiene residuo disponible o biomasa a bajo costo, por ejemplo generada en la misma planta productiva.

Sin embargo, es importante hacer notar que la factibilidad de estos proyectos se ve afectada por el alto costo que tienen las tecnologías disponibles en el mercado para la incineración de BCS. Ya que de acuerdo con el análisis que se realizó, una caldera de biomasa cuesta aproximadamente de 8 a 10 veces más de lo que cuesta una caldera de combustible fósil (gas natural o combustóleo)

Sin embargo, aun cuando el costo de la caldera es sumamente alto se tiene una recuperación de la inversión menor a 4 años, considerando solamente como ingreso la diferencia entre el costo de generación con BCS y combustible fósil. Esta recuperación de inversión es altamente sensible al costo del combustibnle ya que con un incremento en su precio el tiempo de recuperación disminuye considerablemente.

Con respecto a las emisiones, si bien en algunos casos como en el caso del pellet de pino las emisiones de NOx son mayores que las de gas natural, estas se pueden disminuir con sistemas adecuados y las de $\mathrm{CO}_{2}$ son nulas por tratarse de biomasa.

Finalmente, en comparación con el combustóleo, las emisiones son menores en cualquier caso y el proyecto es aún más rentable.

\section{Sugerencias}

Se observa que es importante realizar un análisis de sensibilidad con respecto al costo, tanto del combustible fósil, como del BCS. Asimismo, es importante realizar el análisis con otro tipo de tecnologías de combustión, con el fin de disminuir el costo de amortización y mejorar la factibilidad de los proyectos. 


\section{Agradecimientos}

El presente trabajo se enmarca en los resultados de las etapas 3 y 4 del proyecto Cluster de Biocombustibles sólidos auspiciado por el fondo de Sustentabilidad Energética SENERCONACYT.

\section{Referencias}

Alves R., Perrella J., (2004). Energetic and Exergetic Analysis in Firewood Boiler., Revista Ciencia y Tecnología V. 12, No. 23, pp. 15-24.

Demirbas A. (2005). Potential applications of renewable energy sources, biomass combustion problems in boiler power systems and combustion related environmentalissues. Prog Energy Combust Sci 2005:31-92.

Demirbas A. (2001). Biomass resource facilities and biomass conversion processing for fuels and chemicals. Energy Conversion and Management Volume 42, Issue 11, 1357-1378.

García, I. M. (2019). Reflexiones sobre la economía colaborativa y el capitalismo de las emociones: nuevos retos en el análisis del conflicto Capital-Vida. Lan harremanak: Revista de relaciones laborales, (41), 6.

Guinée, J. (2016). Life Cycle Sustainability Assessment: What Is It and What Are Its Challenges? En R. Clift, \& A. Druckman (Edits.), Taking Stock of Industrial Ecology (págs. 45-68). Springer Open.

Heijungs, R. (2010). Ecodesign - Carbon Footprint - Life Cycle Assessment - Life Cycle Sustainability Analysis. Environmental and Climate Technologies, 4, 42-46.

IPCC, 2006. "2006 IPCC Guidelines for National Greenhouse Gas Inventories", volume 2. [Chapter 2 - Stationary combustion)

IEA (International Energy Agency), World Energy Outlook (2018). Recuperado de: https://ethz.ch/content/dam/ethz/specialinterest/mavt/process-engineering/separationprocesses-laboratory-

dam/documents/education/ccs\%20notes/World \%20Energy\%20Outlook\%202018.pdf.

(Consulado 9 de mayo del 2019).
ISO. (2006). ISO 14040:2006(es). Recuperado de International Organization for Standardization:

https://www.iso.org/obp/ui\#iso:std:iso:14040:e d-2:v1:es

Johansson L., Leckner, Gustavsson L., Cooperc D, Tullin C, Potter A. (2004). Emission characteristics of modern and old-type residential boilers fired with wood logs and wood pellets. Atmospheric Environment 38 : 4183-4195

Keenan J. y Maguire M. (1983). Cost Model of Industrial Fluidized Bed Boiler. J. Energy Eng. 1983,109 (2): 113-126.

Koh MP, Hoi WK (2003). Sustainable biomass production for energy in Malaysia. Biomass Bioenergy 2003;25.

Oregon. (2010). Biomass Energy. Recuperado el el 1 de mayo de 2010 de: http://www.oregon.gov/ENERGY/RENEW/Bio mass/BiomassHome.shtml.

PEMEX. (2018). Precio al público de productos petrolíferos. Obtenido de indicadores petroleros:

http://www.pemex.com/ri/Publicaciones/Indica dores\%20Petroleros/epublico_esp.pdf.

Rodriguez, J. M. F., Noriega, L. H. O., Orozco, I. H., \& Ortega, A. T. (2019). Análisis de Ciclo de Vida para la producción de biodiesel derivado de palma de aceite caso colombiano. Publicaciones e Investigación, 1.

Samudra L., y Molina (2004). M. CÁLCULO DE EMISIONES DE CONTAMINACIÓN ATMOSFÉRICA POR USO DE COMBUSTIBLES FÓSILES EN EL SECTOR ELÉCTRICO MEXICANO, Integraterd Programo Urban, Regional and Global Air Pollution.

SE (2017), Balance Nacional de Energía, Secretaría de Energía. Recuperado el 9 de mayo del 2019 de: https://www.gob.mx/cms/uploads/attachment/fi le/414843/Balance_Nacional_de_Energ_a_201 7.pdf 
Shen X., R. Kommalapati, Huque Z. (2015). The Comparative Life Cycle Assessment of Power Generation from Lignocellulosic Biomass, Sustainability, vol. 7 , no. 10, pp. 12974-12987.

UNEP. (2011). Towards a Life Cycle Sustainability Assessment: Making Informed choices of products. Paris: UNEP.

Vakkilainen E., Kuparinen K, Heinimö J. (2013) Larger Industrial Users of Energy Biomass, IEA BIOENERGY 2013. Recuperado el 4 de mayo del 2019 de: http://task40.ieabioenergy.com/wp459 content/uploads/2013/09/t40-large-industrialbiomass-users.pdf

Walker D. A. (2009), Biofuels, facts, fantasy and feasibility, J Appl Phycol (2009) 21:509517. 